\title{
Stellar and Planetary Characterization of the Ross 128 Exoplanetary System from APOGEE Spectra
}

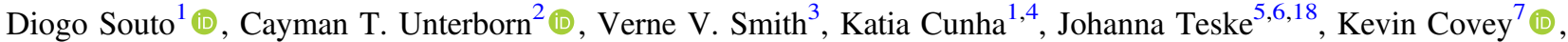 \\ Bárbara Rojas-Ayala $^{8}$, D. A. García-Hernández ${ }^{9,10}$, Keivan Stassun ${ }^{11,12}$ (iD, Olga Zamora ${ }^{9,10}$, Thomas Masseron ${ }^{9,10}$, \\ J. A. Johnson ${ }^{13}$ (D), Steven R. Majewski ${ }^{14}$ (D), Henrik Jönsson ${ }^{15}$ (D), Steven Gilhool ${ }^{16}$ (iD), Cullen Blake ${ }^{16}$ (iD), and Felipe Santana ${ }^{17}$ (D) \\ ${ }^{1}$ Observatório Nacional, Rua General José Cristino, 77, 20921-400 São Cristóvão, Rio de Janeiro, RJ, Brazil; souto@on.br, diogodusouto@gmail.com \\ ${ }^{2}$ School of Earth and Space Exploration, Arizona State University, Tempe, AZ 85287, USA \\ ${ }^{3}$ National Optical Astronomy Observatory, 950 North Cherry Avenue, Tucson, AZ 85719, USA \\ ${ }^{4}$ Steward Observatory, University of Arizona, 933 North Cherry Avenue, Tucson, AZ 85721-0065, USA \\ 5 Department of Terrestrial Magnetism, Carnegie Institution for Science, Washington, DC 20015, USA \\ ${ }^{6}$ The Observatories of the Carnegie Institution for Science, 813 Santa Barbara Street, Pasadena, CA 91101, USA \\ ${ }^{7}$ Department of Physics \& Astronomy, Western Washington University, Bellingham, WA 98225, USA \\ ${ }^{8}$ Departamento de Ciencias Fisicas, Universidad Andres Bello, Fernandez Concha 700, Las Condes, Santiago, Chile \\ ${ }^{9}$ Instituto de Astrofísica de Canarias, E-38205 La Laguna, Tenerife, Spain \\ ${ }^{10}$ Departamento de Astrofísica, Universidad de La Laguna, E-38206 La Laguna, Tenerife, Spain \\ ${ }^{11}$ Department of Physics and Astronomy, Vanderbilt University, 6301 Stevenson Center Ln., Nashville, TN 37235, USA \\ 12 Department of Physics, Fisk University, 1000 17th Ave. N., Nashville, TN 37208, USA \\ ${ }^{13}$ Department of Astronomy, The Ohio State University, Columbus, OH 43210, USA \\ ${ }^{14}$ Department of Astronomy, University of Virginia, Charlottesville, VA 22904-4325, USA \\ ${ }^{15}$ Lund Observatory, Department of Astronomy and Theoretical Physics, Lund University, Box 43, SE-221 00 Lund, Sweden \\ ${ }^{16}$ Department of Physics and Astronomy, University of Pennsylvania, 209 S. 33rd Street, Philadelphia, PA 19104, USA \\ ${ }^{17}$ Universidad de Chile, Av. Libertador Bernardo OHiggins 1058, Santiago De Chile, Chile \\ Received 2018 May 11; revised 2018 May 27; accepted 2018 May 28; published 2018 June 13
}

\begin{abstract}
The first detailed chemical abundance analysis of the M-dwarf (M4.0) exoplanet-hosting star Ross 128 is presented here, based upon near-infrared $(1.5-1.7 \mu \mathrm{m})$, high-resolution $(R \sim 22,500)$ spectra from the SDSS Apache Point Galactic Evolution Experiment survey. We determined precise atmospheric parameters $T_{\text {eff }}=3231 \pm 100 \mathrm{~K}$, $\log g=4.96 \pm 0.11$ dex and chemical abundances of eight elements $(\mathrm{C}, \mathrm{O}, \mathrm{Mg}, \mathrm{Al}, \mathrm{K}, \mathrm{Ca}$, Ti, and $\mathrm{Fe})$, finding Ross 128 to have near solar metallicity $([\mathrm{Fe} / \mathrm{H}]=+0.03 \pm 0.09 \mathrm{dex})$. The derived results were obtained via spectral synthesis (1D LTE) adopting both MARCS and PHOENIX model atmospheres; stellar parameters and chemical abundances derived from the different adopted models do not show significant offsets. Mass-radius modeling of Ross $128 \mathrm{~b}$ indicates that it lies below the pure-rock composition curve, suggesting that it contains a mixture of rock and iron, with the relative amounts of each set by the ratio of Fe/Mg. If Ross $128 \mathrm{~b}$ formed with a subsolar Si abundance, and assuming the planet's composition matches that of the host star, it likely has a larger core size relative to the Earth despite this producing a planet with a Si/Mg abundance ratio $~ 34 \%$ greater than the Sun. The derived planetary parameters-insolation flux $\left(S_{\text {Earth }}=1.79 \pm 0.26\right)$ and equilibrium temperature $\left(T_{\text {eq }}=294 \pm 10 \mathrm{~K}\right)$ - support previous findings that Ross $128 \mathrm{~b}$ is a temperate exoplanet in the inner edge of the habitable zone.
\end{abstract}

Key words: stars: abundances - infrared: stars - planet-star interactions - planetary systems - stars: fundamental parameters - stars: low-mass

\section{Introduction}

Nearby M dwarfs likely provide some of the best opportunities for detecting and characterizing potentially "Earth-like" exoplanets in the near future. M dwarfs produce larger observational signatures from low-mass exoplanets through both the radial velocity (RV) and transit methods (Charbonneau \& Deming 2007; Shields et al. 2016), making it easier to discover Earth-size or Earth-mass exoplanets orbiting these stars. Discoveries of Earth-size or Earth-mass exoplanets have become common around low-mass stars thanks to the efforts of high-cadence radial velocity programs, as well as the Kepler mission (Batalha et al. 2013). Proxima Cen b and the recent announcement of Ross $128 \mathrm{~b}$ are examples of RV-detected exoplanets (Anglada-Escudé et al. 2016, Bonfils et al. 2017), while TRAPPIST-1, Kepler-138, and Kepler-186 (Quintana et al. 2014; Jontof-Hutter et al. 2015; Gillon et al.

\footnotetext{
${ }^{18}$ Hubble Fellow.
}

2017; Souto et al. 2017) are examples of transiting exoplanet systems with cool M-dwarf host stars.

An approach to study the exoplanet composition, albeit an indirect one, is the analysis of the individual host star. This method can, for example, provide measurements of a star's C and $\mathrm{O}$ abundance, which play a role in the ice and gas chemistry in protoplanetary disks, as well as $\mathrm{Mg}, \mathrm{Fe}$, and $\mathrm{Si}$ abundances, that potentially control a rocky planet's core to mantle mass ratios (Bond et al. 2010; Delgado Mena et al. 2010; Thiabaud et al. 2015; Dorn et al. 2017; Santos et al. 2017, and Unterborn \& Panero 2017). Until recently, detailed abundance measurements of M dwarfs were lacking, due in part to the difficulty of obtaining high-S/N, high-resolution spectra, as well as strong molecular absorption from species such as $\mathrm{TiO}$ in the optical or $\mathrm{H}_{2} \mathrm{O}$ in the near-infrared (Allard et al. 2013).

The previous work of Souto et al. (2017) demonstrated that effective temperatures and detailed individual abundances of 13 elements can be measured from near-infrared (NIR) H-band 
high-resolution Apache Point Galactic Evolution Experiment (APOGEE; Majewski et al. 2017) spectra of warm M dwarfs ( $T_{\text {eff }} \sim 3900 \mathrm{~K}$; see also Schmidt et al. 2016). Also using highresolution spectra, Önehag et al. (2012) and Lindgren \& Heiter (2017) have shown that stellar metallicities can be studied from J-band spectra. Most studies, however, use photometric calibrations to determine M-dwarf stellar parameters, e.g., $T_{\text {eff }}, \log g$, and mass (Delfosse et al. 2000; Bonfils et al. 2005; Mann et al. 2015) and low-resolution NIR spectroscopy to determine M-dwarf metallicities $([\mathrm{Fe} / \mathrm{H}]$ ) from equivalent widths in the $\mathrm{K}$ band (Rojas-Ayala et al. 2012). These methods provide a good estimate of the stellar parameters and metallicity; in the context of exoplanet studies, accurate values for $T_{\text {eff }}$ and $R_{\star}$ are needed in order to better constrain the exoplanet properties (e.g., insolation, equilibrium temperature). In addition, precise host star abundance measurements for particular elements can help to constrain the composition of the initial refractory materials that build rocky exoplanets.

In this work, we perform the first detailed abundance analysis for Ross 128, a cool M-dwarf (M4.0) exoplanet host. We use $R \sim 22,500 \mathrm{H}$-band APOGEE spectra to derive precise atmospheric parameters and chemical abundances of eight elements adopting a similar methodology as Souto et al. (2017), but extending the analysis to the cool M-dwarf regime. Such parameters are needed to better characterize the mass $\left(M \sin (i) \sim 1.35 M_{\oplus}\right)$ of the 9.9 day period exoplanet around Ross 128 , the second closest (3.37 pc) terrestrial-mass planet, recently discovered by Bonfils et al. (2017).

\section{Observations and Spectrum Synthesis}

The APOGEE survey (Majewski et al. 2017; see also Blanton et al. 2017; Zasowski et al. 2017) observes primarily red giants, but has also observed $\mathrm{M}$ dwarfs in the solar neighborhood to fill missing fibers or as part of ancillary projects. With APOGEE NIR spectral coverage $(\lambda 15,150-17,000 \AA)$ and high-resolution spectroscopic capabilities (Gunn et al. 2006; Wilson et al. 2010), the APOGEE spectrograph has turned out to be an excellent instrument for detailed studies of $\mathrm{M}$ dwarfs (see Souto et al. 2017).

The APOGEE spectra of Ross 128 (HIP 57548; GJ 447; $2 \mathrm{M} 11474440+0048164)$ were obtained with the fiber feed to the NMSU $1.0 \mathrm{~m}$ telescope at APO. We use the processed spectrum of Ross 128 from the DR14 pipeline (Nidever et al. 2015; Abolfathi et al. 2018) representing the combination of two individual observations both obtained on 2014 January 28. The resulting $\mathrm{S} / \mathrm{N}$ (per pixel) in the combined spectrum is 230 .

As a first step in the analysis we conducted a careful identification of the main spectral features in the APOGEE spectrum of Ross 128, given that this is a much cooler M dwarf than those previously analyzed in Souto et al. (2017). Figure 1 displays the molecular and atomic lines identified; in this $T_{\text {eff }}$ regime most of the APOGEE spectrum is dominated by $\mathrm{H}_{2} \mathrm{O}$ or $\mathrm{FeH}$ lines but some atomic lines of $\mathrm{Fe} \mathrm{I}, \mathrm{Mg} \mathrm{I}, \mathrm{Ca} \mathrm{I}$, and $\mathrm{Al}$ I are also seen. We performed a line-by-line manual abundance analysis to determine the atmospheric parameters of Ross 128 (Section 2.1) and individual chemical abundances of the elements $\mathrm{C}, \mathrm{O}, \mathrm{Mg}, \mathrm{Al}, \mathrm{K}, \mathrm{Ca}, \mathrm{Ti}$, and $\mathrm{Fe}$. The adopted transitions in the line-by-line abundance analysis (a total of 86 lines) are indicated with black vertical tick marks in Figure 1. Figure 1 also shows the best-fit synthetic spectra obtained from this analysis, as discussed below.
A spectrum synthesis analysis is required to analyze cool star (M-dwarf) spectra, rather than the equivalent-width method, due to molecular blends that blanket their spectra. In this work, we adopted 1D plane-parallel LTE models from MARCS and BT-Settl PHOENIX (Gustafsson et al. 2008; Allard et al. 2013). The synthetic spectra were computed with the Turbospectrum code (Alvarez \& Plez 1998; Plez 2012) using the modified version of the APOGEE line list (Shetrone et al. 2015; Souto et al. 2017) that takes into account transitions of FeH. The synthetic spectra were broadened with a Gaussian profile corresponding to the APOGEE resolution (FWHM $\sim 0.73 \AA$ ). We derived a $v \sin i \leqslant 8 \mathrm{~km} \mathrm{~s}^{-1}$ for Ross 128 and note that this $v \sin i$ value is at the limit of what can be resolved from APOGEE spectra Gilhool et al. (2018). In all calculations, we adopted a microturbulent velocity of $\xi=1.00 \mathrm{~km} \mathrm{~s}^{-1}$. The best-fit synthetic spectra were obtained by defining the pseudocontinuum of the observed spectra over each window analyzed, and the derived abundances are the value best fitting the observed spectra from a $\chi 2$-minimization method.

\subsection{Effective Temperature and Surface Gravity}

The effective temperature ( $\left.T_{\text {eff }}\right)$ and surface gravity $(\log g$ ) values of Ross 128 were obtained using a similar methodology as discussed in Souto et al. (2017). That previous study used the $\mathrm{OH}$ and $\mathrm{H}_{2} \mathrm{O}$ lines as oxygen abundance indicators to define the effective temperature. Here, we add $\mathrm{Fe} \mathrm{I}$ and $\mathrm{FeH}$ as $\mathrm{Fe}$ abundance indicators and use the combination of oxygen and iron abundances from $\mathrm{OH}, \mathrm{H}_{2} \mathrm{O}, \mathrm{Fe} \mathrm{I}$, and $\mathrm{FeH}$ for deriving both the effective temperature and the surface gravity for Ross 128 .

Figure 2 shows the derived oxygen and iron abundances as functions of $T_{\text {eff }}$ and $\log g$ for both MARCS and PHOENIX model atmospheres. The effective temperature and $\log g$ are defined by the agreement between the different abundance indicators (crossing points). The derived effective temperature using the $T_{\text {eff }}-A(\mathrm{O})$ pair (top left panel) is quite similar to the one from the $T_{\text {eff }}-A(\mathrm{Fe})$ pair (bottom left panel), with a $T_{\text {eff }}$ difference of only $\sim 5 \mathrm{~K}$; a similar effective temperature is derived using either family of model atmosphere. The same is true for $\log g$, although with slightly larger differences $(\sim 0.02-0.08 \mathrm{dex})$. These results indicate that good consistency can be obtained between the different abundance indicators.

The stellar parameters and individual abundances derived from MARCS and PHOENIX models are very similar (Tables 1 and 2). We derive $T_{\text {eff }}=3231 / 3223 \pm 100 \mathrm{~K}, \quad \log g=$ $4.96 / 4.89 \pm 0.11 \mathrm{dex}$, and $[\mathrm{Fe} / \mathrm{H}]=+0.03 /+0.04 \pm 0.09$ (MARCS/PHOENIX). The uncertainties in $T_{\text {eff }}$ and $\log g$ were estimated by allowing the $\mathrm{Fe}$ abundances from $\mathrm{Fe} \mathrm{I}$ and $\mathrm{FeH}$ lines and oxygen abundances from $\mathrm{H}_{2} \mathrm{O}$ and $\mathrm{OH}$ lines to differ by 0.1 dex. The values from Rojas-Ayala et al. (2012) displayed in Table 1 were obtained using the same techniques as Muirhead et al. (2014). We adopt the $V-J$ and $r-J$ colors to determine the $T_{\text {eff }}$ from Mann et al. (2015). The derived surface gravity $(\log g=4.96)$ agrees well with the $\log g$ obtained from the physical relation $(\log g=5.09)$ assuming $M_{\star}=0.168 \pm$ $0.017 M_{\odot}$ from Mann et al. (2015), $L=0.00367 L_{\odot}$ from Gaia DR2 (Gaia Collaboration et al. 2018), and the $T_{\text {eff }}$ of this work using the MARCS model, where $T_{\text {eff } \odot}=5772 \mathrm{~K}$ and $\log g_{\odot}=$ 4.438 were adopted. Table 2 presents the abundance results, the standard deviation of the mean abundances from the adopted lines (std), and the abundance uncertainties for each species $(\sigma)$ to changes in the atmospheric parameters (computed following Souto et al. 2017). 

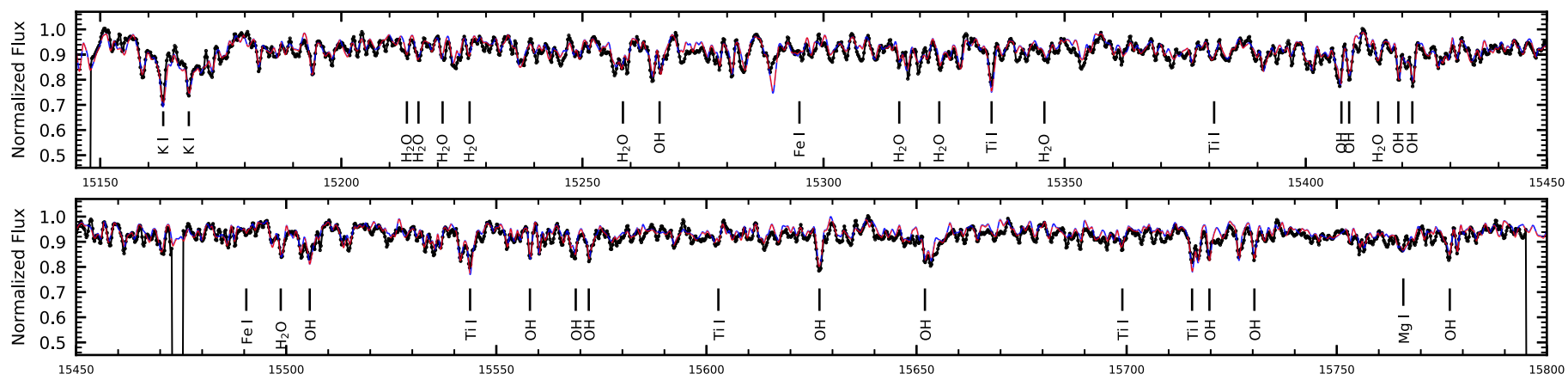

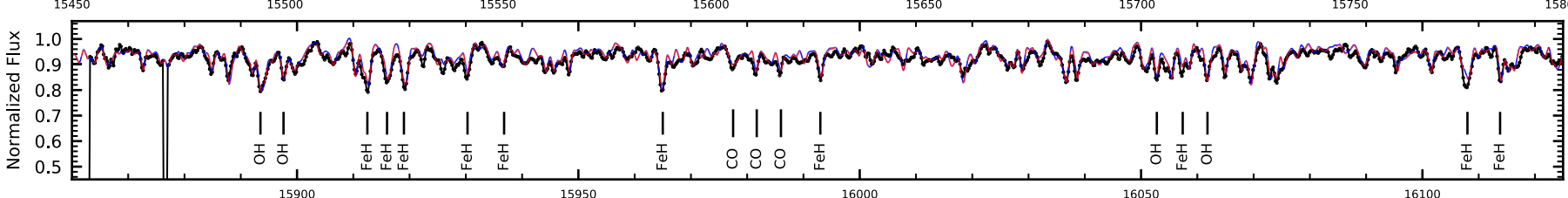
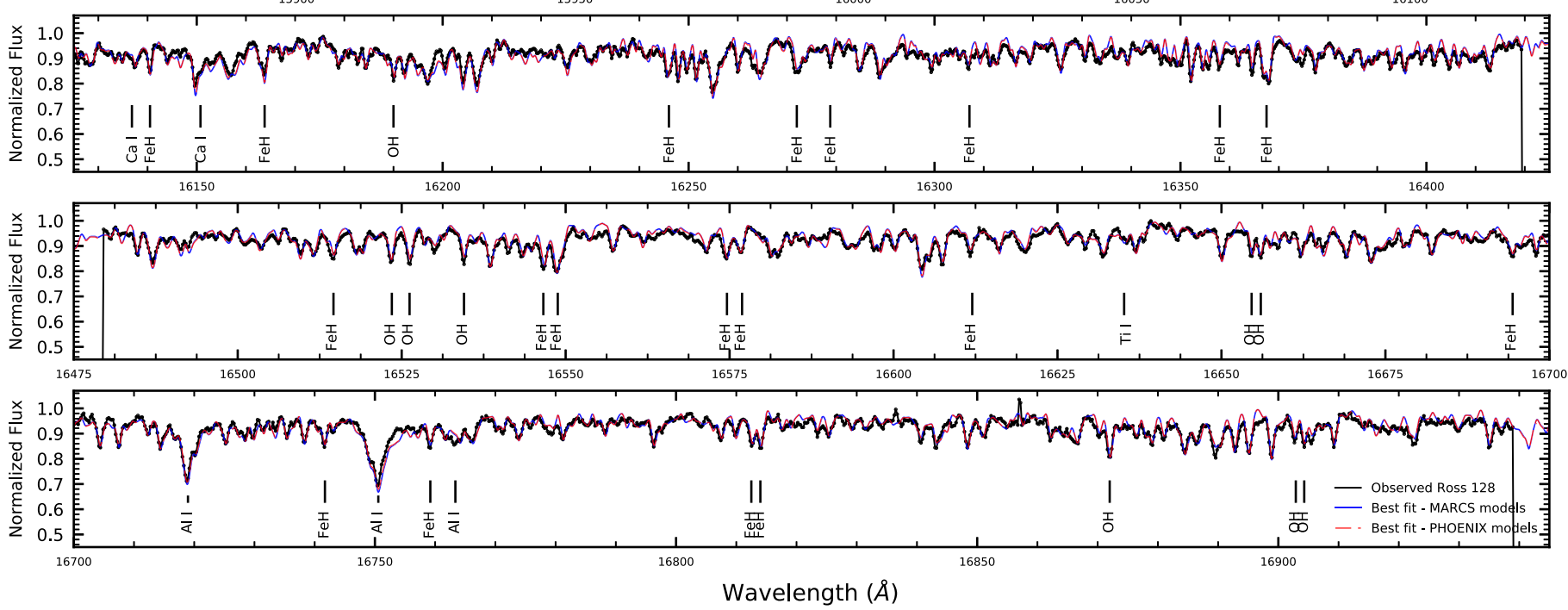

Figure 1. Observed spectra (black) are shown superimposed over the best-fit MARCS (blue) and PHOENIX (red) synthetic spectra using the results from Tables 1 and 2. The lines or features used for the abundance analysis are labeled and indicated with black ticks.

\section{Discussion}

In the following discussion, we adopt the abundance results derived with the MARCS model atmospheres, keeping in mind, however, that the results computed with PHOENIX models are very similar. The derived abundances of Ross 128 are close to solar for all elements, with the exception of $\mathrm{Ti}$ and to a lesser degree $\mathrm{Mg}$ (within the uncertainties). For $\mathrm{Mg}$, we derive $[\mathrm{Mg} / \mathrm{H}]=-0.10 \pm 0.13 \mathrm{dex}$ from one neutral $\mathrm{Mg} \mathrm{I}$ line ( $\lambda 15765.842 \AA)$, while for the other alpha-elements, calcium and oxygen, we find just slightly subsolar abundances $([\mathrm{Ca} / \mathrm{H}]=$ $-0.01 \pm 0.03$ and $[\mathrm{O} / \mathrm{H}]=-0.02 \pm 0.06)$. For $\mathrm{Al}$ we obtain $[\mathrm{Al} / \mathrm{H}]=-0.02$ from one $\mathrm{Al} \mathrm{I}$ line at $\lambda 16763 \AA$; the other two Al I lines ( $\lambda 16718$ and $16750 \AA$ ) present in the APOGEE spectra of Ross 128 are too strong and were not used in our analysis (see also the discussion in Nordlander \& Lind 2017 for non-LTE effects in $\mathrm{Al}$ ). For $\mathrm{Ti}$ we obtain $[\mathrm{Ti} / \mathrm{H}]=-0.17$ from five Ti I lines with large scatter in the abundance results; the standard deviation of the mean is 0.21 dex indicating that the $\mathrm{Ti}$ abundance is uncertain to some degree. Non-LTE effects might be relevant for the TiI H-band lines, but these have not been investigated here nor included in our calculations. We also note that the results for Ti for the APOGEE DR14 red giants present some issues as discussed in Souto et al. (2016) and H. Jönsson et al. (2018, in preparation).

\subsection{Star-Planet Connection}

One of the goals in studying individual abundances of exoplanet host stars is to try to infer the composition of the rocky exoplanets that orbit them. Certain abundance ratios (e.g., $\mathrm{C} / \mathrm{O}$ and $\mathrm{Mg} / \mathrm{Si}$ ) play an important role in the chemistry of exoplanet formation in the disk and may also provide firstorder information on the structure and mineralogy of the resulting rocky planets (e.g., Bond et al. 2010; Unterborn \& Panero 2017). In this work, we derive $\mathrm{C} / \mathrm{O}=0.60 \pm 0.04$ for Ross 128. While this is quite similar to solar $(\mathrm{C} / \mathrm{O}=0.54$; Asplund et al. 2005), it is below the value where refractory carbon is expected to condense and drastically affect the condensation temperatures of the refractory, rocky planetbuilding elements $\mathrm{Mg}, \mathrm{Si}$, and $\mathrm{Fe}(\mathrm{C} / \mathrm{O}=0.8-1.0$; Lodders 2003). The stellar $\mathrm{Mg} / \mathrm{Si}$ abundance ratio may affect the resulting silicate mineralogy of Ross $128 \mathrm{~b}$, although it is not possible to derive $\mathrm{Si}$ abundances in Ross 128 as the $\mathrm{Si}$ I lines become severely blended with $\mathrm{H}_{2} \mathrm{O}$ or $\mathrm{FeH}$. The $\mathrm{Fe} / \mathrm{Mg}$ ratio, however, can affect the relative fraction of core-to-mantle and 

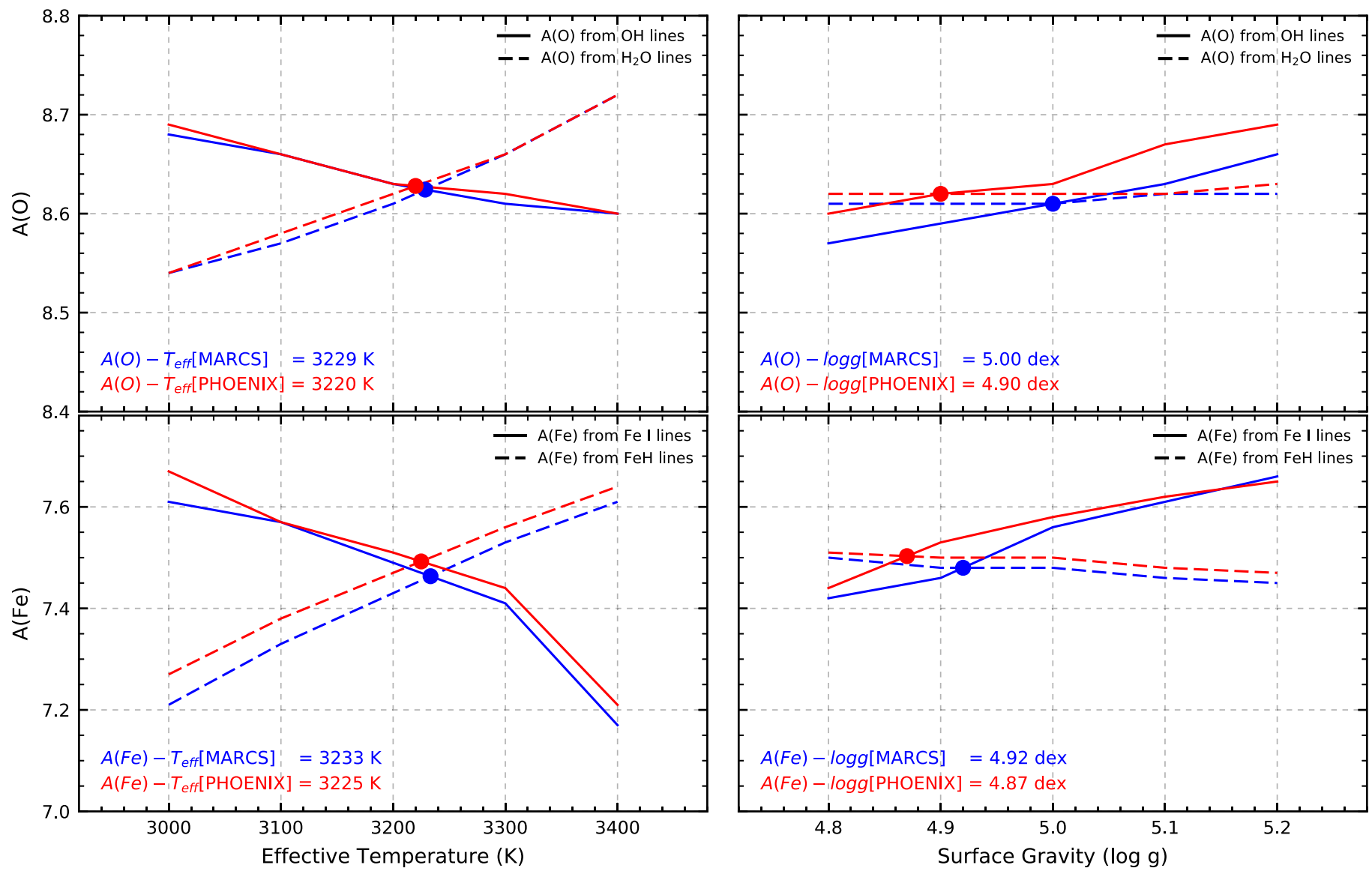

Figure 2. Diagrams illustrating the derivation of atmospheric parameters. The left panels display the $T_{\mathrm{eff}}-A(\mathrm{O})$ and $T_{\mathrm{eff}}-A(\mathrm{Fe})$ curves and the right panels the $\log g-A(\mathrm{O})$ and $\log g-A(\mathrm{Fe})$ pairs. In all panels, the derived abundances from $\mathrm{Fe} \mathrm{I}$ and $\mathrm{OH}$ lines are shown as solid lines, while those from $\mathrm{FeH}$ and $\mathrm{H}_{2} \mathrm{O}$ as dashed lines. The different colors (blue and red) indicate results derived from the adopted models, MARCS and PHOENIX, respectively.

Table 1

Atmospheric Parameters

\begin{tabular}{lccccc}
\hline \hline & $T_{\text {eff }}$ & $\log g$ & $\xi$ & {$[\mathrm{Fe} / \mathrm{H}]$} & {$[\mathrm{O} / \mathrm{H}]$} \\
\hline MARCS & $3231 \pm 100$ & $4.96 \pm 0.11$ & 1.00 & +0.02 & -0.03 \\
PHOENIX & $3223 \pm 100$ & $4.89 \pm 0.11$ & 1.00 & +0.03 & -0.02 \\
Rojas-Ayala & $2986 \pm 50$ & $\cdots$ & $\cdots$ & +0.03 & $\cdots$ \\
$\quad$ et al. (2012) & & & & & \\
Mann & $3192 \pm 64$ & 5.08 & $\ldots$ & -0.02 & $\cdots$ \\
$\quad$ et al. (2015) & & & & & \\
\hline
\end{tabular}

provide some broad constraints on the possible structures of Ross $128 b$.

While both mass and radius are not available for Ross $128 \mathrm{~b}$, we can estimate its radius given its observed minimum mass and assuming the stellar composition of the host star is a proxy for that of the planet. We calculate the range of radii possible for Ross 128b using the ExoPlex software package (Unterborn et al. 2018) for all masses above the minimum mass of Ross 128b (1.35 $M_{\oplus}$; Bonfils et al. 2017). Models were run assuming a two-layer model with a liquid core and silicate mantle (no atmosphere). We increase the input mass until a likely radius of $1.5 R_{\oplus}$ was achieved, roughly the point where planets are not expected be gas-rich mini-Neptunes as opposed to rock and iron-dominated super-Earths (Figure 3, left). ExoPlex conserves the relative molar ratios for the dominant rocky planetbuilding elements assuming all $\mathrm{Fe}$ is present in the core with no light elements (e.g., Si, O, H) present in the core. Adopting our
MARCS abundances we get the molar ratios $\mathrm{Fe} / \mathrm{Mg}=1.12$, $\mathrm{Ca} / \mathrm{Mg}=0.09$, and $\mathrm{Al} / \mathrm{Mg}=0.08$, where the Earth and solar values are 0.90/0.07/0.09 (McDonough 2003) and 0.83/0.06/ 0.08 (Lodders 2003), respectively.

Changes in the relative planet's core size have a large effect on its bulk density (Unterborn et al. 2016), and we thus consider our $\mathrm{Fe}$ abundance uncertainty: $0.88 \leqslant \mathrm{Fe} / \mathrm{Mg} \leqslant 1.3$. We also varied $\mathrm{Si} / \mathrm{Mg}$ between the extremes of 0.5 and 2 (Solar $\mathrm{Si} / \mathrm{Mg}$ is 0.97$)$. This range of $\mathrm{Si} / \mathrm{Mg}$ represents an average difference in radius for a given input mass of roughly $0.05 R_{\oplus}$ (Figure 3, left panel). However, because the molar mass of $\mathrm{MgO}$ is $1 / 3$ smaller than $\mathrm{SiO}_{2}$ (our chosen mantle oxides), this range of $\mathrm{Si} / \mathrm{Mg}$ represents core mass fractions between 0.44 and 0.27 (Earth is 0.33 ) for $\mathrm{Si} / \mathrm{Mg}$ of 0.5 and 2, respectively. For this model, only if Ross $128 \mathrm{~b}$ had a supersolar $\mathrm{Si} / \mathrm{Mg}$ of 1.3 would it match the Earth in its core mass fraction, which equates to Ross 128 having a $\mathrm{Si}$ abundance of roughly solar $(\mathrm{A}(\mathrm{Si})=7.54)$. That is to say while the molar ratio of $\mathrm{Fe} / \mathrm{Mg}$ is conserved, the mass ratio of $\mathrm{Fe} / \mathrm{Mg}$ in our model is not and depends on the chosen $\mathrm{Mg} / \mathrm{Si}$. This means that if Ross $128 \mathrm{~b}$ 's composition mimics that of its host star and a subsolar $\mathrm{Si} / \mathrm{Mg}$, it will have a larger relative core size than Earth, despite having roughly solar iron abundance. This is due to the relative ratio of Fe to $\mathrm{Mg}$ being greater than in the Sun and Earth, combined with the density of liquid $\mathrm{Fe}$ being greater than that of magnesium silicates (e.g., rock dominated). We note that the uncertainty in the derived radii is about the size of the red curve in Figure 3 (left panel). 
Table 2

Individual Abundances

\begin{tabular}{|c|c|c|c|c|c|c|c|c|}
\hline & $A(\mathrm{X})$ & $\begin{array}{c}\text { MARCS } \\
{[\mathrm{X} / \mathrm{H}]}\end{array}$ & std & $A(\mathrm{X})$ & $\begin{array}{c}\text { PHOENIX } \\
{[\mathrm{X} / \mathrm{H}]}\end{array}$ & std & Number of Lines & $\sigma$ \\
\hline Fe I & 7.48 & 0.03 & 0.02 & 7.49 & 0.04 & 0.02 & 2 & 0.09 \\
\hline $\mathrm{FeH}$ & 7.45 & 0.00 & 0.07 & 7.46 & 0.01 & 0.08 & 30 & 0.08 \\
\hline $\mathrm{C}$ & 8.41 & 0.02 & 0.01 & 8.41 & 0.02 & 0.01 & 3 & 0.02 \\
\hline $\mathrm{OH}$ & 8.62 & -0.04 & 0.02 & 8.63 & -0.03 & 0.02 & 29 & 0.06 \\
\hline $\mathrm{H}_{2} \mathrm{O}$ & 8.64 & -0.02 & 0.02 & 8.64 & -0.02 & 0.03 & 10 & 0.08 \\
\hline $\mathrm{Mg}$ & 7.43 & -0.10 & $\cdots$ & 7.48 & -0.05 & $\cdots$ & 1 & 0.13 \\
\hline $\mathrm{Al}$ & 6.35 & -0.02 & $\cdots$ & 6.36 & -0.01 & $\cdots$ & 1 & 0.10 \\
\hline K & 5.03 & -0.05 & 0.03 & 5.06 & -0.02 & 0.03 & 2 & 0.04 \\
\hline $\mathrm{Ca}$ & 6.36 & -0.01 & 0.02 & 6.38 & 0.01 & 0.03 & 2 & 0.03 \\
\hline $\mathrm{Ti}$ & 4.73 & -0.17 & 0.21 & 4.77 & -0.13 & 0.20 & 6 & 0.09 \\
\hline
\end{tabular}

Our calculated Ross $128 \mathrm{~b}$ radii-under the assumption that the stellar chemical abundances are also those in the planet-all lie below the $100 \%$ rock composition curve of Zeng et al. (2016), i.e., it contains a mixture of rock and Fe, with the relative amounts of each set by $\mathrm{Fe} / \mathrm{Mg}$. Given our compositional constraints, these calculated radii represent the minimum radius of Ross $128 \mathrm{~b}$ in the absence of collisional stripping of silicate relative to iron (see also Benz et al. 1988; Marcus et al. 2010). This means that the addition of light elements to the core or the presence of surface water or an atmosphere will only lower the planet density, causing a radius increase for the same mass. The Ross $128 \mathrm{~b}$ relatively Fe-rich composition may affect its geodynamo (Gaidos et al. 2010; Driscoll \& Olson 2011), although the geodynamic and geochemical consequences of Fe-rich planets are not well explored.

With the results presented here, we can study some Ross $128 \mathrm{~b}$ fundamental habitability parameters such as insolation flux $\left(S_{\text {Earth}}\right.$; flux of energy the exoplanet receives from its host star) and equilibrium temperature $\left(T_{\text {eq }}\right)$. Bonfils et al. (2017) predict that Ross $128 \mathrm{~b}$ should have an insolation flux $\left(S_{\text {Earth }}\right)$ of 1.38. Adopting our MARCS $T_{\text {eff }}$, the stellar radius $\left(R_{\star}=\right.$ $0.209 \pm 0.002 R_{\odot}$; very similar to Mann et al. 2015) derived by us from the spectral energy distribution (Stassun et al. 2017) and adopting the Bonfils et al. (2017) semimajor axis $(\mathrm{SMA}=0.049 \mathrm{au})$, we derive $S_{\text {Earth }}=1.79 \pm 0.26$ for Ross $128 \mathrm{~b}$, which is consistent with Bonfils et al. (2017). The $T_{\text {eq }}$ of an exoplanet is a function of the stellar $T_{\text {eff }}$, radius, the SMA of the planet and its albedo. We obtain for Ross $128 \mathrm{~b}$ a $T_{\text {eq }}=294 \pm 10 \mathrm{~K}$ for an Earth-like albedo (0.306). Adopting a Venus- and Mars-like albedo (0.77 and 0.25), we derive a $T_{\text {eq }}$ of $223 \pm 0.08$ and $299 \pm 11 \mathrm{~K}$, respectively. Our results support the claim of Bonfils et al. (2017) that Ross 128b is a temperate exoplanet in the inner edge of the habitable zone. However, this is not to say that Ross $128 \mathrm{~b}$ is a "Exo-Earth." Geologic factors unexplored in Bonfils et al. (2017) such as the planet's likelihood to produce continental crust, the weathering rates of key nutrients into ocean basins, or the presence of a long-term magnetic field could produce a planet decidedly not at all "Earth-like" or habitable due to differences in its composition and thermal history. Furthermore, other aspects of the M dwarf's stellar activity and its effect on the retention of any atmosphere and potential habitability should be studied, although we find no evidence of activity in the Ross 128 spectra.

In Figure 3, we use the NASA Exoplanet Archive to study the planetary density (left panel), the metallicity distribution of the host stars as well as the insolation flux and the planet equilibrium temperature as a function of the distance of the systems to the Sun (right panels). We split the sample of known exoplanets into four groups based on the mass-radius bulkcomposition curves from Zeng et al. (2016). The planets with density $(\rho)<1$ were tagged as Cold $\mathrm{H}_{2} / \mathrm{He}$, where $1<\rho<4$ have a predominant $\mathrm{H}_{2} \mathrm{O}$ atmosphere, the rock-dominated exoplanets have $4<\rho<10$, and those with $\rho>10$ are tagged as Fe-rich exoplanets; we did not take density errors into account for these rough categories. To give particular attention to the $\mathrm{M}$ dwarfs hosting exoplanets, we split the sample into stars with $T_{\text {eff }}<4100 \mathrm{~K}$ (squared symbols) and $T_{\text {eff }}>4100 \mathrm{~K}$ (circled symbols) using the same cuts in density. In Figure 3 (left panel), likely rocky exoplanets are highlighted and the gray shadow region indicates the potential regime for Earth-like exoplanets (with radii from 0 to $1.5 R_{\oplus}$ and masses from 0 to $\left.5 M_{\oplus}\right)$. The estimated radii for Ross $128 \mathrm{~b}$ are displayed as a solid red line. The size of the red line represents the typical uncertainty in the estimated radii.

We show in the right panel of Figure 3 the metallicity $([\mathrm{Fe} / \mathrm{H}])$ distance $(\mathrm{pc})$ distribution of the host stars, with the exoplanet insolation flux (Figure 3, top right panel) and equilibrium temperature (Figure 3, bottom right panel) represented by color bars. We compute the exoplanet insolation flux and the equilibrium temperature using the $T_{\text {eff }}, R_{\star}$, and SMA from the NASA Exoplanet Archive with an Earth-like albedo $(30 \%)$ for all exoplanets. For the M dwarfs without $R_{\star}$ in the database, we adopt Mann et al. (2015) calibrations to determine the stellar radius. With a green shadow, we highlight the region with $d \leqslant 20 \mathrm{pc}$, calling attention to the opportunity provided by the nearby M-dwarf exoplanetary systems. The symbols follow the same notation of Figure 3 (left panel), with the addition of small-dot systems where the exoplanet density is not measured. The $\mathrm{M}$ dwarfs closer than $20 \mathrm{pc}$ that host planets without measured densities are shown as upside down triangles. Ross $128 \mathrm{~b}$ is also presented as blue crosses. We leave the water and gaseous planets as background symbols. From Figure 3 (top right panel) the exoplanets with the lower degree of insolation flux orbit M-dwarf stars (yellow squares and triangles). In contrast, the rocky exoplanets around solar-like stars tend to receive much more flux, generally 1000 times more than the Earth.

In summary, our precise spectroscopic atmospheric parameters and individual abundances have allowed us to use theoretical models to study the potential interior composition of Ross 128b. Assuming Ross $128 \mathrm{~b}$ formed with the same composition as its host star we calculate its mineralogy, structure, and thus its mass. Our model assumes no atmosphere 

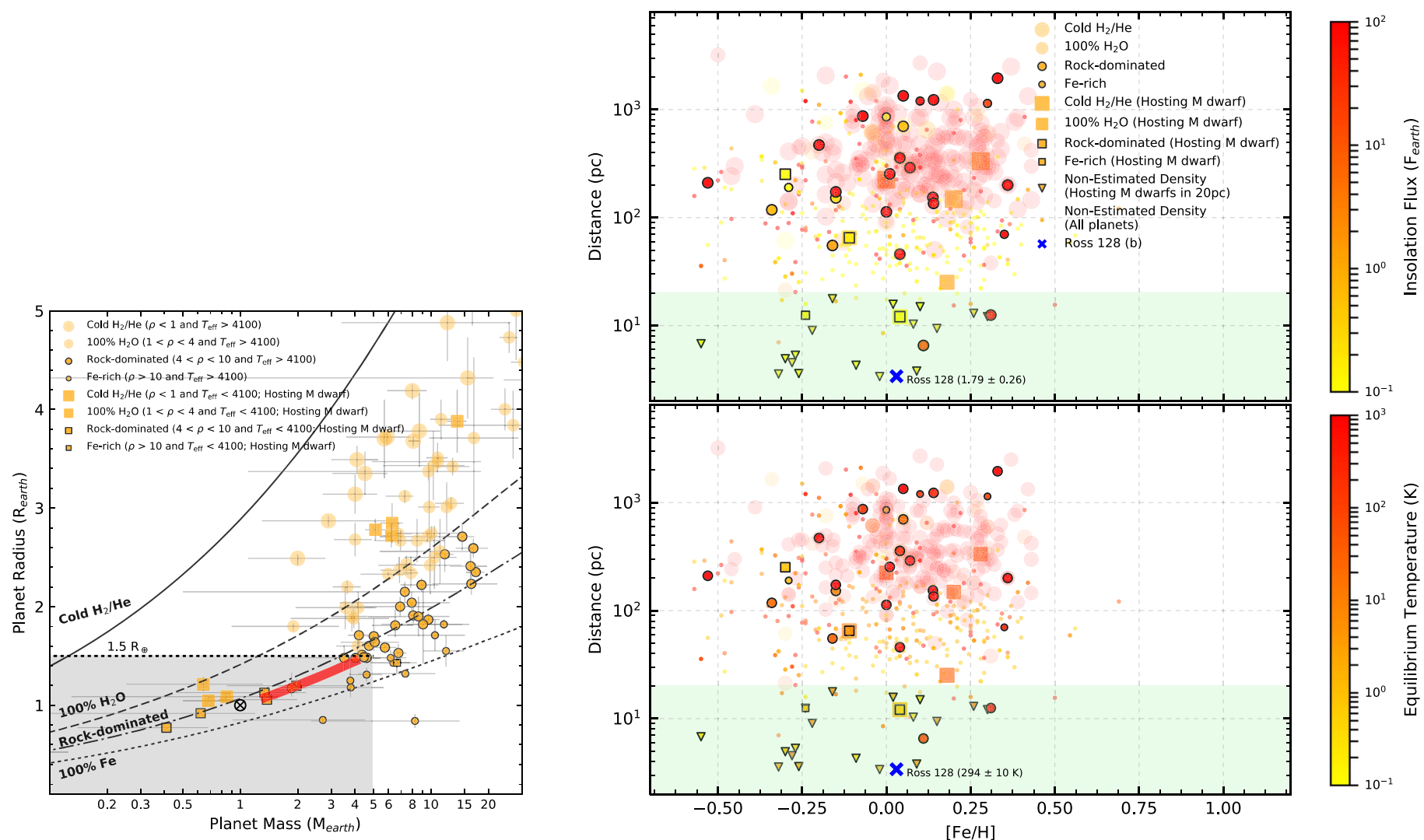

Figure 3. Left panel: the exoplanet mass-radius diagram using the planetary composition based on Zeng et al. (2016) relations. Exoplanets around M dwarfs are shown with orange squares, and the other stars hosting exoplanets are shown with orange circles. Right panels: the metallicity-distance diagram; in the top panel, we plot the computed insolation flux at the planet's distance from the star, and in the bottom panel, we adopt the planet's equilibrium temperature in the color bar. The symbols follow the same notation from the left panel with the addition of upside down triangles for the M dwarfs that are closer than $20 \mathrm{pc}$ from the Sun and host exoplanets without density measurements. We use dots to indicate the stars hosting exoplanets without density measured.

is present, however, because the addition of this layer (or the addition of light elements to the core) would decrease the density for a given radius, our calculated masses represent the maximum mass of Ross $128 \mathrm{~b}$ in the absence of any mantle stripping due to large impacts. In this scenario, if Ross 128 is also depleted in Si relative to Solar we calculate Ross $128 \mathrm{~b}$ would have a relatively larger core than the Earth, regardless of the mantle chemistry. It is a likely scenario given the relative depletion of alpha-elements in Ross 128 . The derived planetary parameters $S_{\text {Earth }}=1.79 \pm 0.26$ and $T_{\mathrm{eq}}=294 \pm 10 \mathrm{~K}$ support the Bonfils et al. (2017) findings that Ross 128b is a temperate exoplanet in the inner edge of the habitable zone.

K.C. and V.S. acknowledge that their work here is supported, in part, by the National Aeronautics and Space Administration under Grant 16-XRP16 $\backslash 2-0004$, issued through the Astrophysics Division of the Science Mission Directorate. D.A.G.H., O.Z., and T.M. acknowledge support provided by the Spanish Ministry of Economy and Competitiveness (MINECO) under grant AYA-2017-88254-P. S.R.M. acknowledges support from NSF grant AST-1616636. B.R-A acknowledges the support from CONICYT PAI/CONCURSO NACIONAL INSERCIÒN EN LA ACADEMIA, CONVOCATORIA 201579150050. H.J. acknowledges support from the Crafoord Foundation and Stiftelsen Olle Engkvist Byggmästare. J.K.T. acknowledges support for this work provided by NASA through Hubble Fellowship grant HST-HF2-51399.001 awarded by the Space Telescope Science Institute, which is operated by the
Association of Universities for Research in Astronomy, Inc., for NASA, under contract NAS5-26555.

This research has made use of the NASA Exoplanet Archive, which is operated by the California Institute of Technology, under contract with the National Aeronautics and Space Administration under the Exoplanet Exploration Program.

Funding for the Sloan Digital Sky Survey IV has been provided by the Alfred P. Sloan Foundation, the U.S. Department of Energy Office of Science, and the Participating Institutions. SDSS-IV acknowledges support and resources from the Center for High-Performance Computing at the University of Utah. The SDSS web site is www.sdss.org.

SDSS-IV is managed by the Astrophysical Research consortium for the Participating Institutions of the SDSS Collaboration including the Brazilian Participation Group, the Carnegie Institution for Science, Carnegie Mellon University, the Chilean Participation Group, the French Participation Group, HarvardSmithsonian Center for Astrophysics, Instituto de Astrofísica de Canarias, The Johns Hopkins University, Kavli Institute for the Physics and Mathematics of the Universe (IPMU)/University of Tokyo, Lawrence Berkeley National Laboratory, Leibniz Institut für Astrophysik Potsdam (AIP), Max-Planck-Institut für Astronomie (MPIA Heidelberg), Max-Planck-Institut für Astrophysik (MPA Garching), Max-Planck-Institut für Extraterrestrische Physik (MPE), National Astronomical Observatory of China, New Mexico State University, New York University, University of Notre Dame, Observatório Nacional/MCTI, The Ohio State University, Pennsylvania State University, Shanghai Astronomical Observatory, United Kingdom Participation 
Group, Universidad Nacional Autónoma de México, University of Arizona, University of Colorado Boulder, University of Oxford, University of Portsmouth, University of Utah, University of Virginia, University of Washington, University of Wisconsin, Vanderbilt University, and Yale University.

Facilities: Sloan, NASA Exoplanet Archive.

Software: ExoPlex (Unterborn et al. 2018), Turbospectrum (Alvarez \& Plez 1998; Plez 2012), MARCS (Gustafsson et al. 2008), PHOENIX BT-Settl (Allard et al. 2013).

\section{ORCID iDs}

Diogo Souto (iD https://orcid.org/0000-0002-7883-5425 Cayman T. Unterborn (iD https://orcid.org/0000-00018991-3110

Kevin Covey (iD https://orcid.org/0000-0001-6914-7797

Keivan Stassun (D) https://orcid.org/0000-0002-3481-9052

J. A. Johnson (iD https://orcid.org/0000-0001-7258-1834

Steven R. Majewski (i) https://orcid.org/0000-0003-

2025-3147

Henrik Jönsson (1D https://orcid.org/0000-0002-4912-8609

Steven Gilhool (D) https://orcid.org/0000-0002-3230-3052

Cullen Blake (ib https://orcid.org/0000-0002-6096-1749

Felipe Santana (D) https://orcid.org/0000-0002-4023-7649

\section{References}

Abolfathi, B., Aguado, D. S., Aguilar, G., et al. 2018, ApJS, 235, 42 Allard, F., Homeier, D., Freytag, B., et al. 2013, MSAIS, 24, 128 Alvarez, R., \& Plez, B. 1998, A\&A, 330, 1109

Anglada-Escudé, G., Amado, P. J., Barnes, J., et al. 2016, Natur, 536, 437 Asplund, M., Grevesse, N., \& Sauval, A. J. 2005, in ASP Conf. Ser. 336, Cosmic Abundances as Records of Stellar Evolution and Nucleosynthesis, ed. T. G. Barnes III \& F. N. Bash (San Francisco, CA: ASP), 25 Batalha, N. M., Rowe, J. F., Bryson, S. T., et al. 2013, ApJS, 204, 24 Benz, W., Slattery, W. L., \& Cameron, A. G. W. 1988, Icar, 74, 516 Blanton, M. R., Bershady, M. A., Abolfathi, B., et al. 2017, AJ, 154, 28 Bond, J. C., O'Brien, D. P., \& Lauretta, D. S. 2010, ApJ, 715, 1050 Bonfils, X., Astudillo-Defru, N., Díaz, R., et al. 2017, arXiv:1711.06177 Bonfils, X., Delfosse, X., Udry, S., et al. 2005, A\&A, 442, 635 Charbonneau, D., \& Deming, D. 2007, arXiv:0706.1047

Delfosse, X., Forveille, T., Ségransan, D., et al. 2000, A\&A, 364, 217
Delgado Mena, E., Israelian, G., González Hernández, J. I., et al. 2010, ApJ, 725, 2349

Dorn, C., Hinkel, N. R., \& Venturini, J. 2017, A\&A, 597, A38

Driscoll, P., \& Olson, P. 2011, Icar, 213, 12

Gaia Collaboration, Brown, A. G. A., Vallenari, A., et al. 2018, arXiv:1804. 09365

Gaidos, E., Conrad, C. P., Manga, M., \& Hernlund, J. 2010, ApJ, 718, 596

Gilhool, S. H., Blake, C. H., Terrien, R. C., et al. 2018, AJ, 155, 38

Gillon, M., Triaud, A. H. M. J., Demory, B.-O., et al. 2017, Natur, 542, 456

Gunn, J. E., Siegmund, W. A., Mannery, E. J., et al. 2006, AJ, 131, 2332

Gustafsson, B., Edvardsson, B., Eriksson, K., et al. 2008, A\&A, 486, 951

Jontof-Hutter, D., Rowe, J. F., Lissauer, J. J., Fabrycky, D. C., \& Ford, E. B. 2015, Natur, 522, 321

Lindgren, S., \& Heiter, U. 2017, A\&A, 604, A97

Lodders, K. 2003, ApJ, 591, 1220

Majewski, S. R., Schiavon, R. P., Frinchaboy, P. M., et al. 2017, AJ, 154, 94

Mann, A. W., Feiden, G. A., Gaidos, E., Boyajian, T., \& von Braun, K. 2015 , ApJ, 804, 64

Marcus, R. A., Sasselov, D., Hernquist, L., \& Stewart, S. T. 2010, ApJL, 712, L73

McDonough, W. F. 2003, TrGeo, 2, 568

Muirhead, P. S., Becker, J., Feiden, G. A., et al. 2014, ApJS, 213, 5

Nidever, D. L., Holtzman, J. A., Allende Prieto, C., et al. 2015, AJ, 150, 173

Nordlander, T., \& Lind, K. 2017, A\&A, 607, A75

Önehag, A., Heiter, U., Gustafsson, B., et al. 2012, A\&A, 542, A33

Plez, B. 2012, Turbospectrum: Code for Spectral Synthesis, Astrophysics Source Code Library, ascl:1205.004

Quintana, E. V., Barclay, T., Raymond, S. N., et al. 2014, Sci, 344, 277

Rojas-Ayala, B., Covey, K. R., Muirhead, P. S., \& Lloyd, J. P. 2012, ApJ, 748, 93

Santos, N. C., Adibekyan, V., Dorn, C., et al. 2017, A\&A, 608, A94

Schmidt, S. J., Wagoner, E. L., Johnson, J. A., et al. 2016, MNRAS, 460, 2611

Shetrone, M., Bizyaev, D., Lawler, J. E., et al. 2015, ApJS, 221, 24

Shields, A. L., Ballard, S., \& Johnson, J. A. 2016, PhR, 663, 1

Souto, D., Cunha, K., García-Hernández, D. A., et al. 2017, ApJ, 835, 239

Souto, D., Cunha, K., Smith, V., et al. 2016, ApJ, 830, 35

Stassun, K. G., Collins, K. A., \& Gaudi, B. S. 2017, AJ, 153, 136

Thiabaud, A., Marboeuf, U., Alibert, Y., Leya, I., \& Mezger, K. 2015, A\&A, 580, A30

Unterborn, C. T., Desch, S. J., Hinkel, N. R., \& Lorenzo, A. 2018, NatAs, 2, 297

Unterborn, C. T., Dismukes, E. E., \& Panero, W. R. 2016, ApJ, 819, 32

Unterborn, C. T., \& Panero, W. R. 2017, ApJ, 845, 61

Wilson, J. C., Hearty, F., Skrutskie, M. F., et al. 2010, Proc. SPIE, 7735, $77351 \mathrm{C}$

Zasowski, G., Cohen, R. E., Chojnowski, S. D., et al. 2017, AJ, 154, 198

Zeng, L., Sasselov, D. D., \& Jacobsen, S. B. 2016, ApJ, 819, 127 